\title{
ACL-reconstructed and ACL-deficient individuals show differentiated trunk, hip, and knee kinematics during vertical hops more than 20 years post-injury
}

\author{
Jonas L. Markström ${ }^{1} \cdot$ Eva Tengman $^{1}$ - Charlotte K. Häger ${ }^{1}$
}

Received: 7 July 2016 / Accepted: 20 March 2017 / Published online: 23 March 2017

(c) The Author(s) 2017. This article is an open access publication

\begin{abstract}
Purpose Little is known regarding movement strategies in the long term following injury of the anterior cruciate ligament (ACL), and even less about comparisons of reconstructed and deficient knees in relation to healthy controls. The present purpose was to compare trunk, hip, and knee kinematics during a one-leg vertical hop (VH) 20 years post-ACL injury between persons treated with surgery and physiotherapy $\left(\mathrm{ACL}_{\mathrm{R}}\right)$, solely physiotherapy $\left(\mathrm{ACL}_{\mathrm{PT}}\right)$, and controls (CTRL). Between-leg kinematic differences within groups were also investigated.

Methods Sixty-six persons who suffered unilateral ACL injury on average $23 \pm 2$ years ago $\left(32 \mathrm{ACL}_{\mathrm{R}}, 34 \mathrm{ACL}_{\mathrm{PT}}\right)$ and 33 controls performed the VH. Peak trunk, hip, and knee angles during Take-off and Landing phases recorded with a 3D motion capture system were analysed with multivariate statistics.

Results Significant group effects during both Take-off and Landing were found, with $\mathrm{ACL}_{\mathrm{PT}}$ differing from CTRL in Take-off with a combination of less knee flexion and knee internal rotation, and from both $\mathrm{ACL}_{\mathrm{R}}$ and CTRL in Landing with less hip and knee flexion, knee internal rotation, and greater hip adduction. $\mathrm{ACL}_{\mathrm{R}}$ also presented different kinematics to $\mathrm{ACL}_{\mathrm{PT}}$ and CTRL in Take-off with a combination of greater trunk flexion, hip flexion, hip internal
\end{abstract}

Jonas L. Markström

jonas.markstrom@umu.se

Eva Tengman

eva.tengman@umu.se

Charlotte K. Häger

charlotte.hager@umu.se

1 Department of Community Medicine and Rehabilitation, Physiotherapy, Umeå University, 90187 Umeå, Sweden rotation, and less knee abduction, and in Landing with greater trunk flexion and hip internal rotation. Further, different kinematics and hop height were found between legs within groups in both Take-off and Landing for both ACL groups, but not for CTRL.

Conclusion Different kinematics for the injured leg for both ACL groups compared to CTRL and between treatment groups, as well as between legs within treatment groups, indicate long-term consequences of injury. Compensatory mechanisms for knee protection seem to prevail over time irrespective of initial treatment, possibly increasing the risk of re-injury and triggering the development of osteoarthritis. Detailed investigation of movement strategies during the $\mathrm{VH}$ provides important information and a more comprehensive evaluation of knee function than merely hop height. More attention should also be given to the trunk and hip in clinics when evaluating movement strategies after ACL injury.

Level of evidence Prospective cohort study, Level II.

Keywords Anterior cruciate ligament - Treatment · Long-term · Movement strategy $\cdot$ One-leg vertical hop

\section{Introduction}

Anterior cruciate ligament (ACL) injuries are very common in sports and occur mainly in non-contact situations with multidirection knee loading in eccentric movements [12]. Besides its primary function of restraining anterior tibial translation, the ACL simultaneously prevents excessive rotation of the tibia relative to the femur [2]. Kinematic alterations have been reported in the short term post-ACL injury during demanding hop activities $[7,19]$, but have been less investigated in the longer term 
and with inconclusive results [27, 30]. Consequences of such kinematic alterations may include mechanical instability, further triggering the development of osteoarthritis (OA) due to altered loadings in the knee joint and thus may affect quality of life [2].

Human movements are characterized by numerous degrees of freedom for coordination and control with multiple rotations and translations from several joints occurring simultaneously. In kinematic analyses, some of these variables will be related to each other, thus providing a set of $n$ variables that may reflect underlying dimensions [5]. These dimensions may be detected using multivariate analyses, thus providing a more accurate description of consequences after ACL injury than if using univariate methods. Such analyses are warranted also in the long term post-ACL injury during demanding knee tasks related to everyday life in order to fully understand the impact of an injury. A suitable task is the vertical hop $(\mathrm{VH})$, which is commonly used in clinical environments and considered an important dynamic movement in physical activity [23]. The VH has also been evaluated in research and showed the highest overall sensitivity and accuracy of five frequently used hop tests, with very good to excellent reliability for hop height [11]. To our knowledge, there is no study examining reliability of kinematics specifically for the VH. Excellent to good reliability for kinematics has, however, been shown for the similar drop-vertical jump [15] and single-leg landing tasks [1]. Vandenberg et al. [28] recently pointed out the importance of considering kinematics of the hip, rather than only the knee, for ACL-injured persons. Despite a high hop height and acceptable limb symmetry index (LSI) for the knee, there might be a changed combined movement strategy that may be possible to detect and characterize by using kinematics and applying multivariate statistics.

The main aim of the present study was therefore to for the first time present an investigation of combined kinematics for the trunk, hip, and knee in the long term ( $>20$ years on average) post-unilateral ACL injury for persons treated with either surgery and physiotherapy $\left(\mathrm{ACL}_{\mathrm{R}}\right)$ or with solely physiotherapy $\left(\mathrm{ACL}_{\mathrm{PT}}\right)$, and compared to a control group (CTRL), when performing the $\mathrm{VH}$. A second aim was to investigate kinematics between legs within groups. Based on previously reported results of functional tasks in the same study population [10, 27], it was anticipated that $\mathrm{ACL}_{\mathrm{PT}}$ would demonstrate more deviating kinematics compared to CTRL than $\mathrm{ACL}_{\mathrm{R}}$ to compensate for knee instability, mainly by a reduced active range of motion. It would also seem logical that there would be differences for combined movement strategies between the injured and non-injured legs within both treatment groups. Consideration of combined movement strategies is getting increased clinical awareness, but how to estimate this needs further attention.

\section{Materials and methods}

This cross-sectional research programme involved three groups, consisting of two cohorts from 113 individuals who suffered an ACL injury 17-28 years previously and were treated at two separate hospitals using different treatment approaches, and a control group. A subset of 81 participants with ACL injury was eligible for the present study according to the following inclusion criteria: unilateral ACL injury, not having any surgical total hip or knee replacement (prosthesis), no inflammatory or rheumatic disease or neurological pathology. The ACLinjured persons were treated either with physiotherapy in combination with reconstructive surgery $\left(\mathrm{ACL}_{\mathrm{R}}, n=42\right)$ or solely with physiotherapy ( $\mathrm{ACL}_{\mathrm{PT}}, n=39$ ). Eleven persons declined to participate $\left(9 \mathrm{ACL}_{\mathrm{R}}\right.$ and $\left.2 \mathrm{ACL}_{\mathrm{PT}}\right)$ due to time constraints and logistical reasons, resulting in $33 \mathrm{ACL}_{\mathrm{R}}$ and $37 \mathrm{ACL}_{\mathrm{PT}}$ participants. Details of treatments have been presented previously [25]. Briefly, persons in $\mathrm{ACL}_{\mathrm{R}}$ had physiotherapy treatment for three months before surgery, and all persons had a patellar tendon autograft. A knee brace and crutches were used for 14 weeks after surgery followed by functional exercises with progressively increased demands. For persons in $\mathrm{ACL}_{\mathrm{PT}}$, a tailored training programme for functional stability was adapted which aimed to achieve an LSI of over $90 \%$ for strength and functional tests. Radiological knee OA mostly in stage $1-2$, but in some cases up to 4 [14], was detected in $\sim 90 \%$ of the participants in both ACL groups at the time of testing. Kinematics of the knee joint were, however, not affected by their OA in a recent publication [27] and were therefore omitted from the analyses of the current study.

One person from $A C L_{R}$ and three persons from $A C L_{P T}$ were excluded from the analyses due to lost marker data in sensitive parts of the VH. A total of 66 ACL-injured persons were therefore included: $32 \mathrm{ACL}_{\mathrm{R}}$ (12 women) and $34 \mathrm{ACL}_{\mathrm{PT}}$ (13 women). The $\mathrm{ACL}$ was injured during team sports (e.g. soccer, floorball) for 53 persons in contact or non-contact, during individual sports (e.g. downhill skiing) for nine persons, and by accident outside of sports for four persons. The CTRL group consisted of 33 persons matched for age and sex (11 women) with no previous knee injuries and normal results from a clinical knee examination. All participants were given written and oral information, and the participants gave their written informed consent according to the Declaration of Helsinki. 


\section{Test protocol}

Participants first completed the International Physical Activity Questionnaire (IPAQ), Tegner activity scale, Lysholm questionnaire, Knee Injury and Osteoarthritis Outcome Score (KOOS), and Tampa Scale for Kinesiophobia (TSK), as described elsewhere [25]. Tibial anterior translation was measured with KT1000 arthrometer (Medmetric Corporation, San Diego, CA, USA) at an anterior pull force of $30 \mathrm{lb}$. Body height and mass were measured to calculate body mass index (BMI). The test procedure began with a 6-min warm-up on a bicycle ergometer at moderate intensity. The VH was performed barefoot on a custom-built force plate with the participants initially standing upright on one leg with arms held across their chest before hopping vertically upwards as high as possible and landing with the same leg on the force plate while maintaining balance. A successful trial required the participant to stand stable after landing for about $2 \mathrm{~s}$ without putting the contralateral foot down or removing their arms from their chest. Participants had one to three practice hops and then performed three to four trials on each leg. ACL-injured persons started on the non-injured leg, and CTRL started on the dominant leg and then alternate between legs. Quadriceps and hamstrings strength was then assessed but presented in an earlier paper [26]. The dominant leg was defined as the preferred leg for kicking a ball. The same physiotherapist (ET) instructed all participants.

\section{Data collection}

Kinematics were captured at $240 \mathrm{~Hz}$ using a motion capture system with eight cameras (Oqus ${ }^{\circledR}$, Qualisys AB, Gothenburg, Sweden) with markers placed bilaterally on the acromion, clavicle, iliac crest, anterior superior iliac spine, greater trochanters, lateral/medial femoral epicondyles, patellas, tuberositas tibia, fibula head, lateral/medial malleoli, lateral/medial foot, on the sternum, and between the posterior superior iliac spines. Three-marker rigid clusters on thighs and shanks were used in order to reduce soft tissue artefacts and increase reliability and precision [9]. Events used for analyses were set using the custom-made force plate (Department of Biomedical Engineering and Informatics, Umeå University Hospital, Sweden) registering ground reactions at $1200 \mathrm{~Hz}$ synchronized with the motion capture system.

\section{Data analysis}

The highest VH for each participant was used in the statistical analyses. The injured (I) leg of persons in the ACL groups was compared to the non-dominant (ND) leg of CTRL, henceforth referred to as I/ND leg, and the non-injured (NI) leg was compared to the dominant (D) leg, henceforth referred to as NI/D leg. Two phases were investigated: (1) Take-off, defined from peak knee flexion to force signal registration $<10 \mathrm{~N}$, and (2) Landing, defined from force signal registration $>10 \mathrm{~N}$ to peak knee flexion. The outcome variables were peak angles of trunk flexion, hip flexion, hip adduction, hip internal rotation, knee flexion, knee abduction, and knee internal rotation. Trunk flexion was defined as movement of the trunk segment relative to the vertical axis in the global coordinate system, and hip and knee joint angles were defined as movement of the distal segment relative to the proximal. The software Qualisys Track Manager (Qualisys AB, Gothenburg, Sweden, version 2.2) and Visual3D (v.5.02.19, C-Motion Inc. Germantown, MD, USA) were used for data processing. An eight-segment rigid body model consisting of feet, shanks, thighs, pelvis, and trunk was constructed, with joint centre calculations based on a six-degrees-of-freedom model [9]. Hop height was calculated using the centre of mass from this model between normal standing and at the highest point of the VH. Data were filtered at $15 \mathrm{~Hz}$ with a critically damped digital filter before further calculations. The project was approved by the Regional Ethical Review Board in Umeå (Dnr. 08-211 M).

\section{Statistical analyses}

Hop performances within and between groups were investigated with paired $t$ tests and one-way ANOVAs with Bonferroni post hoc tests if significant. Multivariate ANOVAs (MANOVAs) were used to investigate kinematics between groups, and repeated measures MANOVAs were used within subjects. Since BMI differed between treatment groups and CTRL, correlations between BMI and kinematic variables in the sagittal plane were investigated (Pearson), but presented no significant correlations. The effect of BMI as a covariate in MANCOVA was also investigated for the same variables, although with no differences in results. BMI was therefore omitted as a covariate. Significant MANOVAs were investigated with direct discriminant analyses (DISCRIM) [5], with correlations $>0.32$ interpreted. Within-subject contrasts were used for between-leg comparisons. Assumptions of absence of multivariate outliers and multicollinearity, linearity, and homogeneity of variance-covariance were met. A power analysis based on pilot tests including five ACL-injured persons and five controls suggested that 32 persons/group were needed for a power of $80 \%$ to detect a significant difference in knee joint flexion angle between groups with a variance of $10^{\circ}$ and a significance level of 5\% (which was also used in analyses). All statistical analyses were conducted using IBM SPSS (version 22, Armonk, New York, USA). 
Table 1 Background data and hop height for each group

\begin{tabular}{|c|c|c|c|}
\hline & $\begin{array}{l}\mathrm{ACL}_{\mathrm{R}} \\
N=32\end{array}$ & $\begin{array}{l}\mathrm{ACL}_{\mathrm{PT}} \\
N=34\end{array}$ & $\begin{array}{l}\text { CTRL } \\
N=33\end{array}$ \\
\hline BMI & $27.1(3.3)^{\mathrm{a}}$ & $28.7(4.3)^{\mathrm{a}}$ & $24.6(2.5)$ \\
\hline Age (years) & $45.5(4.6)$ & $47.6(5.9)$ & $46.7(5.0)$ \\
\hline Years since ACL injury & $23.8(2.7)$ & $23.1(1.3)$ & - \\
\hline Years since ACL surgery & $20.1(1.5)$ & - & - \\
\hline Anterior translation I/ND-NI/D leg (mm) & $2.0(2.7)^{\mathrm{a}, \mathrm{b}}$ & $4.9(2.9)^{\mathrm{a}}$ & $-0.1(1.1)$ \\
\hline Hop height I/ND leg (m) & $0.20(0.04)^{\mathrm{b}, \mathrm{c}}$ & $0.17(0.03)^{\mathrm{a}, \mathrm{c}}$ & $0.20(0.04)$ \\
\hline Hop height NI/D leg (m) & $0.21(0.04)$ & $0.19(0.04)$ & $0.20(0.03)$ \\
\hline LSI hop height $(\%)$ & $94(13)$ & $91(14)^{\mathrm{a}}$ & $104(23)$ \\
\hline $\mathrm{KOOS}_{\text {SYMPTOMS }}$ (score) & $84(100)^{\mathrm{a}}$ & $75(61)^{\mathrm{a}}$ & $100(7)$ \\
\hline $\operatorname{KOOS}_{\text {PAIN }}$ (score) & $82(58)^{\mathrm{a}}$ & $89(50)^{\mathrm{a}}$ & $100(6)$ \\
\hline $\operatorname{KOOS}_{\mathrm{ADL}}$ (score) & $89(58)^{\mathrm{a}, \mathrm{b}}$ & $98(62)^{\mathrm{a}}$ & $100(0)$ \\
\hline $\mathrm{KOOS}_{\mathrm{SPORT}}$ (score) & $50(100)^{\mathrm{a}, \mathrm{b}}$ & $75(100)^{\mathrm{a}}$ & $100(10)$ \\
\hline $\mathrm{KOOS}_{\mathrm{QOL}}($ score $)$ & $49(22)^{\mathrm{a}, \mathrm{b}}$ & $69(94)^{\mathrm{a}}$ & $100(13)$ \\
\hline TSK (score) & $33(55)$ & $32(27)$ & - \\
\hline IPAQ (score) & $2391(1591)$ & $2254(2045)$ & $2570(2097)$ \\
\hline IPAQ (assigned score) & $2(2)$ & $2(2)$ & $2(2)$ \\
\hline Tegner pre-injury (score) & $9(7)^{\mathrm{b}}$ & $9(6)$ & - \\
\hline Tegner 20 years (score) & $4(4)^{\mathrm{a}}$ & $4(5)^{\mathrm{a}}$ & $6(4)$ \\
\hline Lysholm 20 years (score) & $81(64)^{\mathrm{a}}$ & $73(61)^{\mathrm{a}}$ & $100(0)$ \\
\hline
\end{tabular}

Data presented in mean (SD) with the exception of questionnaires (analysed with nonparametric statistics) with scores presented in median (range). LSI, limb symmetry index being the ratio between I/NI leg for ACL groups and ND/D leg for CTRL; I/ND leg, injured leg for ACL groups and non-dominant leg for CTRL; NI/D leg, non-injured leg for ACL groups and dominant leg for CTRL. For more details of questionnaires and hop performances, see Tengman et al. [25]

a Significant difference observed to CTRL

b Significant difference observed to $\mathrm{ACL}_{\mathrm{PT}}$

c Significant difference observed to the NI leg within groups

\section{Results}

Background data and hop height are presented in Table 1. Both treatment groups had significantly greater laxity for the affected compared to the non-affected leg ( $p<0.001$ for both groups), with $\mathrm{ACL}_{\mathrm{PT}}$ showing a significantly greater difference compared to $\mathrm{ACL}_{\mathrm{R}}(95 \%$ CI of $1.6-4.3 \mathrm{~mm}$ difference, $p<0.001)$. A significant effect of group on hop height for I/ND comparisons was found ( $p=0.007$ ), with $\mathrm{ACL}_{\mathrm{PT}}$ having a lower hop height than both $\mathrm{ACL}_{\mathrm{R}}$ (95\% CI of 0.001-0.05 $\mathrm{m}$ difference, $p=0.04$ ) and CTRL (95\% CI of 0.01-0.05 m difference, $p=0.01$ ), while no differences were shown between $\mathrm{ACL}_{\mathrm{R}}$ and CTRL. Both treatment groups had lower hop height with their injured compared to their non-injured $\operatorname{leg}\left(p=0.006\right.$ for $\mathrm{ACL}_{\mathrm{R}}$ and $p=0.001$ for $\left.\mathrm{ACL}_{\mathrm{PT}}\right)$. Average kinematic angles are presented in Figs. 1 and 2 for Take-Off and Landing phases, respectively. Peak angles for trunk, hip, and knee kinematics in Take-off and Landing for I/ND and NI/D legs are presented in Table 2.

\section{Kinematics for injured/non-dominant leg in Take-off and Landing phases}

Take-off: significant kinematic differences were found for I/ND legs between groups during Take-off $(p<0.001)$. Follow-up analysis with DISCRIM revealed two significant discriminant functions $(p<0.001$ with canonical $R^{2}=0.26$ and $p=0.018$ with canonical $R^{2}=0.15$ ). High correlations were found for knee internal rotation and knee flexion to the first function ( $r=0.63$ and 0.59 , respectively) and for trunk flexion, hip internal rotation, knee abduction, and hip flexion for the second function ( $r=0.59,0.49,0.43$, and 0.40 , respectively). The discriminant function plot in Fig. 3a shows the first function separating $A C L_{P T}$ from CTRL, and the second function separating $A C L_{R}$ from $A C L_{P T}$ and CTRL. That is, $A C L_{P T}$ demonstrated a combination of less internal rotation of the knee (i.e. negative angles in Table 2 denote greater external rotation) and less knee flexion than CTRL, and $A C L_{R}$ demonstrated a combination of greater trunk 

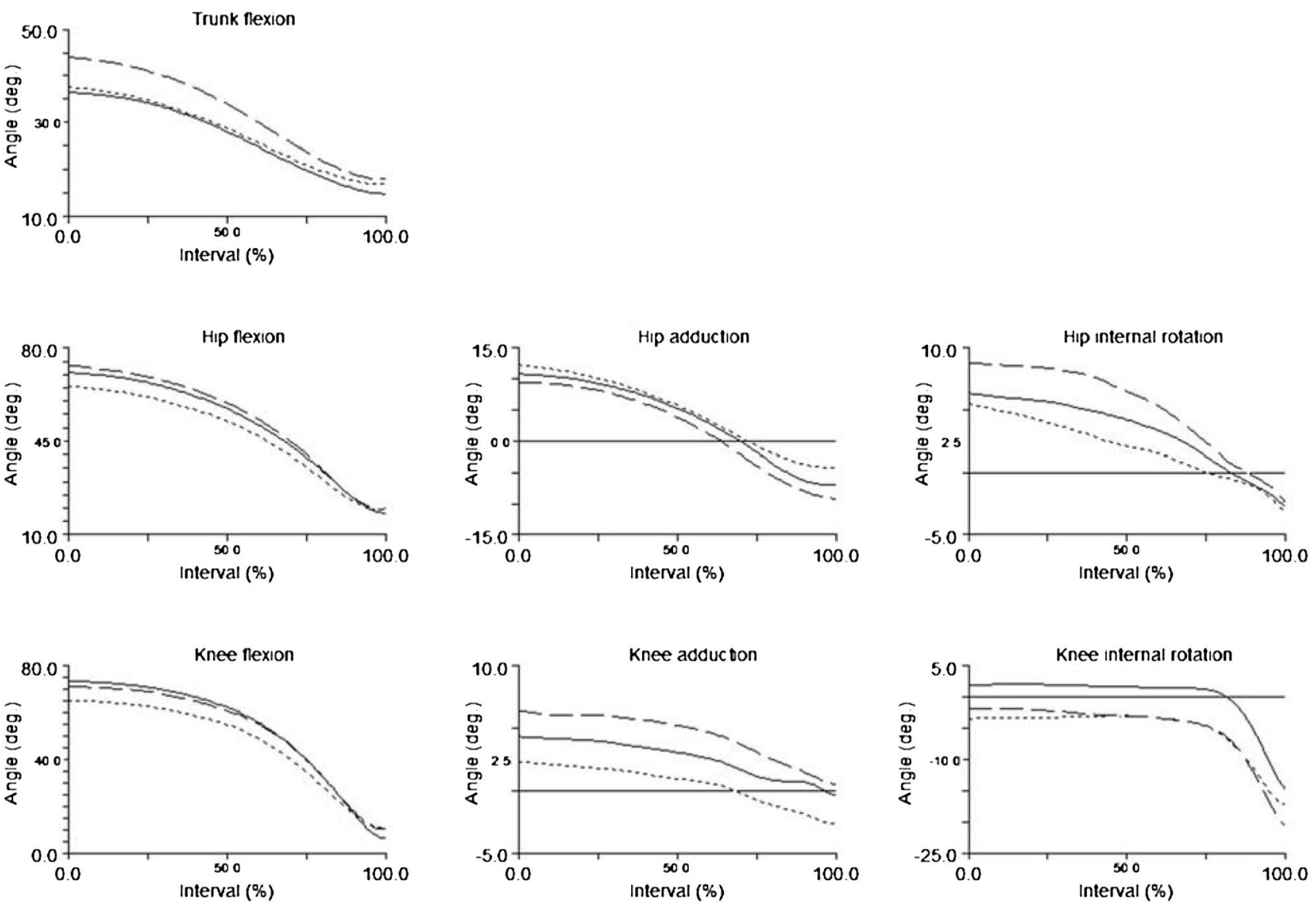

Fig. 1 Ensemble mean trunk, hip, and knee kinematics during the time-normalized Take-off phase of the injured leg for ACL groups, and of the non-dominant leg for CTRL. Flexion, adduction, and inter-

flexion, greater hip internal rotation, less knee abduction, and greater hip flexion than both $\mathrm{ACL}_{\mathrm{PT}}$ and CTRL.

Landing: significant differences were found for I/ND legs between groups during Landing $(p<0.001)$. DISCRIM revealed two significant discriminant functions $(p<0.001$ with canonical $R^{2}=0.28$ and $p=0.001$ with canonical $R^{2}=0.22$ ). High correlations were found for knee flexion, hip flexion, knee internal rotation, and hip adduction to the first function $(r=0.71,0.62,0.57$, and -0.33 , respectively) and for trunk flexion and hip internal rotation for the second function $(r=-0.60$ and -0.35 , respectively). The discriminant function plot in Fig. $3 \mathrm{~b}$ shows the first function separating $\mathrm{ACL}_{\mathrm{PT}}$ from both $\mathrm{ACL}_{\mathrm{R}}$ and CTRL and the second function separating $A C L_{R}$ from both $A C L_{P T}$ and CTRL. Thus, $\mathrm{ACL}_{\mathrm{PT}}$ demonstrated a combination of less knee and hip flexion, less knee internal rotation (i.e. greater external rotation), and greater hip adduction than $\mathrm{ACL}_{\mathrm{R}}$ and CTRL, and $A C L_{R}$ demonstrated a combination of greater trunk flexion and hip internal rotation than both $\mathrm{ACL}_{\mathrm{PT}}$ and CTRL. nal rotation in positive angles. Angle at $0^{\circ}$ is marked with a horizontal line. Each interval on the $Y$-axis is equal to $5^{\circ}$. Dashed line for $\mathrm{ACL}_{\mathrm{R}}$, dotted line for $\mathrm{ACL}_{\mathrm{PT}}$, solid line for $\mathrm{CTRL}$

\section{Kinematics in non-injured/dominant leg in Take-off and Landing phases}

Significant differences were also found for NI/D legs between groups during Take-off ( $p=0.004)$, but not in Landing (n.s.). DISCRIM revealed one significant discriminant function ( $p=0.002$ with canonical $R^{2}=0.27$ ) with high correlations with knee flexion, knee abduction, hip flexion, and hip internal rotation $(r=0.72,0.62,0.52$, and 0.37 , respectively). The discriminant function plot in Fig. $3 c$ shows $A C L_{P T}$ differing from both $A_{C L}$ and CTRL by demonstrating less knee flexion, greater knee abduction, less hip flexion, and less hip internal rotation.

\section{Leg comparisons within groups}

Between-leg comparisons showed significant differences for $\mathrm{ACL}_{\mathrm{R}}$ and $\mathrm{ACL}_{\mathrm{PT}}$ in both Take-off $(p<0.001$ and $p=0.004$, respectively) and Landing ( $p=0.026$ and 

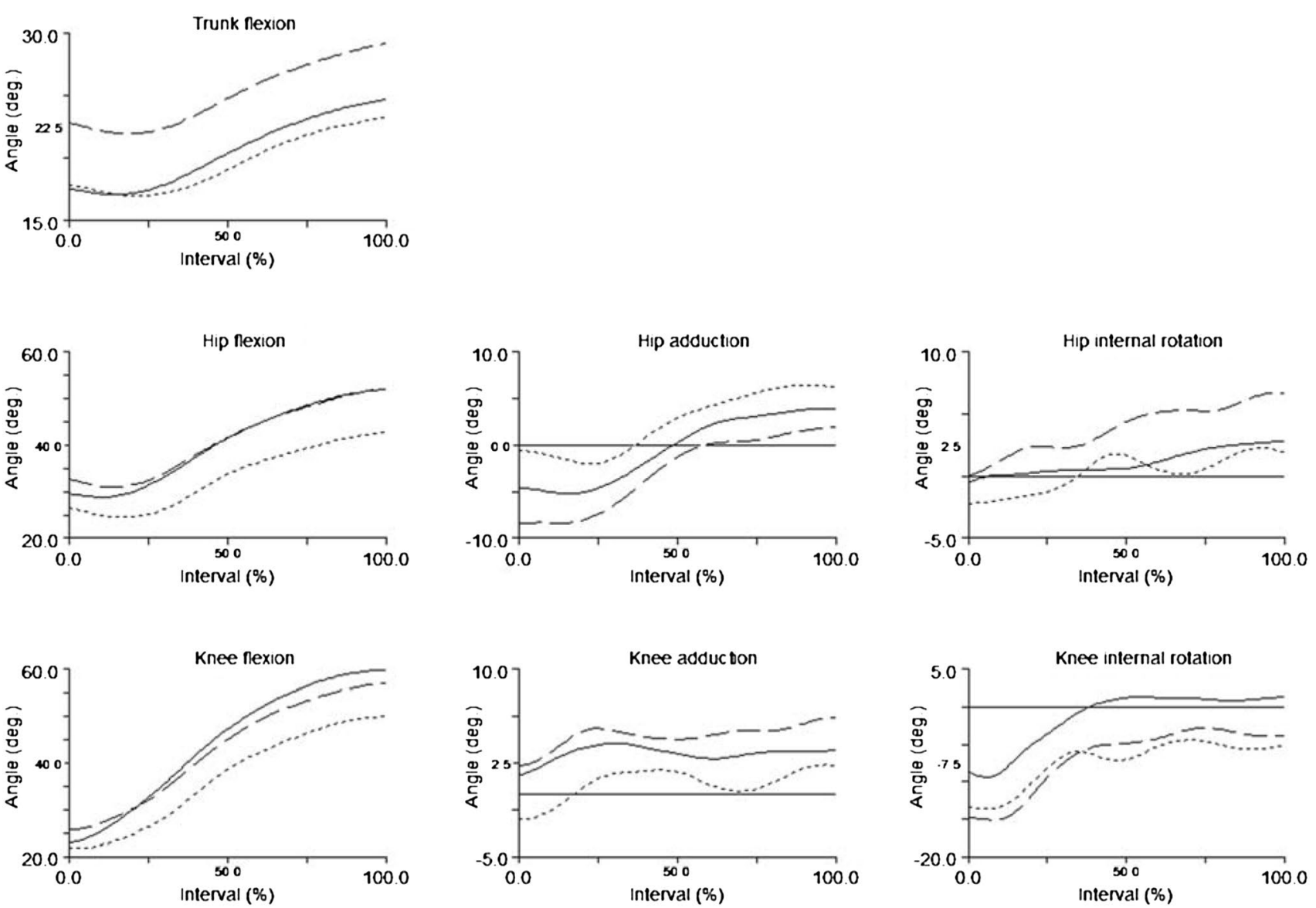

Fig. 2 Ensemble mean trunk, hip, and knee kinematics during the time-normalized Landing phase of the injured leg for ACL groups, and of the non-dominant leg for CTRL. Flexion, adduction, and inter-

0.020 , respectively), but not for CTRL in any of the phases (n.s.). See Table 2 for within-subject contrasts.

\section{Discussion}

The main finding of the present study is that the combined movement strategies of the trunk, hip, and knee differed for the two treatment groups and compared to healthy controls during the $\mathrm{VH}$. More specifically, $\mathrm{ACL}_{\mathrm{PT}}$ demonstrated kinematics characterized by reduced hip flexion, knee flexion, and knee rotation, while $\mathrm{ACL}_{\mathrm{R}}$ presented the greatest trunk flexion, hip flexion, and hip rotation. The greater difference between legs in anterior tibial laxity for $\mathrm{ACL}_{\mathrm{PT}}$ may explain these movement strategies. $\mathrm{ACL}_{\mathrm{PT}}$ also presented lower hop height than $\mathrm{ACL}_{\mathrm{R}}$ and CTRL. Despite this, there were similar scores for the two ACL groups on Tegner activity scale and IPAQ, and higher scores on the KOOS subscales ADL, SPORT, and QOL for $A C L_{P T}$ compared to $A C L_{R}$, indicating that $\mathrm{ACL}_{\mathrm{PT}}$ had not adapted a more restricted nal rotation in positive angles. Angle at $0^{\circ}$ is marked with a horizontal line. Each interval on the $Y$-axis is equal to $5^{\circ}$. Dashed line for $\mathrm{ACL}_{\mathrm{R}}$, dotted line for $\mathrm{ACL}_{\mathrm{PT}}$, solid line for CTRL

movement strategy compared to $\mathrm{ACL}_{\mathrm{R}}$. This may indicate that knee scores alone do not capture the entire picture with regard to knee function evaluation, and thus, motion analysis provides additional value.

In the absence of an ACL, knee stability relies on the remaining static and dynamic stabilizers to restrict the tibia from excessive anterior displacement and rotation. Since the ACL has an oblique medial orientation from the femur to the tibia [2], a ruptured ACL may result in a more internally rotated tibial position during passive motion. Indeed, in knees where the ACL has been removed, an increased internal rotation has been shown during application of an axial tibial force [16]. In the present study, the observed less pronounced knee internal rotation, in fact an externally rotated knee, in combination with less knee flexion in Take-off for $\mathrm{ACL}_{\mathrm{PT}}$ compared to CTRL, may therefore be a knee-protective strategy to avoid positions of internal rotation where give way might occur. The same strategy was found during Landing, although with the addition of less hip flexion and greater hip adduction when compared 
Table 2 Peak angles of trunk, hip, and knee joints presented in mean (SD)

\begin{tabular}{|c|c|c|c|c|}
\hline & \multicolumn{2}{|l|}{ I/ND leg } & \multicolumn{2}{|l|}{ NI/D leg } \\
\hline & TO & LA & TO & LA \\
\hline \multicolumn{5}{|c|}{ Trunk forward flexion $\left({ }^{\circ}\right)$} \\
\hline $\mathrm{ACL}_{\mathrm{R}}$ & $44.2(12.7)^{\mathrm{a}}$ & $30.0(8.9)^{\mathrm{a}}$ & $40.6(11.2)$ & $26.0(9.7)$ \\
\hline $\mathrm{ACL}_{\mathrm{PT}}$ & $37.7(11.0)^{\mathrm{a}}$ & $23.8(7.7)$ & $35.2(11.5)$ & $23.0(9.4)$ \\
\hline CTRL & $39.1(9.6)$ & $23.6(9.9)$ & $36.6(9.4)$ & $24.8(9.7)$ \\
\hline \multicolumn{5}{|c|}{ Hip flexion $\left(^{\circ}\right)$} \\
\hline $\mathrm{ACL}_{\mathrm{R}}$ & $73.3(15.4)$ & $52.44(12.0)$ & $71.7(12.4)$ & $50.1(11.9)$ \\
\hline $\mathrm{ACL}_{\mathrm{PT}}$ & $65.4(16.7)$ & $43.0(11.5)$ & $63.1(15.5)$ & $44.8(13.1)$ \\
\hline CTRL & 70.7 (12.9) & $52.0(10.7)$ & $72.3(12.5)$ & $49.8(10.9)$ \\
\hline \multicolumn{5}{|c|}{ Hip adduction $\left({ }^{\circ}\right)$} \\
\hline $\mathrm{ACL}_{\mathrm{R}}$ & $9.8(6.6)$ & $3.3(7.8)$ & $10.6(6.8)$ & $4.7(6.9)$ \\
\hline $\mathrm{ACL}_{\mathrm{PT}}$ & $12.3(7.5)$ & $7.8(7.6)$ & $10.2(6.9)$ & $5.8(8.7)$ \\
\hline CTRL & $11.0(5.6)$ & $5.5(5.6)$ & $11.0(6.9)$ & $5.6(8.0)$ \\
\hline \multicolumn{5}{|c|}{ Hip internal rotation $\left(^{\circ}\right)$} \\
\hline $\mathrm{ACL}_{\mathrm{R}}$ & $10.6(8.2)^{\mathrm{a}}$ & $10.6(8.1)$ & $6.7(6.4)$ & $7.6(7.0)$ \\
\hline $\mathrm{ACL}_{\mathrm{PT}}$ & $6.6(9.3)^{\mathrm{a}}$ & $6.5(7.8)$ & $2.9(8.4)$ & $4.0(8.5)$ \\
\hline CTRL & $7.6(6.3)$ & $7.5(7.5)$ & $6.1(7.1)$ & $6.8(9.1)$ \\
\hline \multicolumn{5}{|c|}{ Knee flexion $\left(^{\circ}\right)$} \\
\hline $\mathrm{ACL}_{\mathrm{R}}$ & $71.3(10.5)^{\mathrm{a}}$ & $57.0(10.6)$ & $74.3(8.7)$ & $58.1(9.2)$ \\
\hline $\mathrm{ACL}_{\mathrm{PT}}$ & $65.3(9.7)$ & $50.0(9.3)^{\mathrm{a}}$ & $66.6(7.9)$ & $54.7(8.9)$ \\
\hline CTRL & $73.6(8.8)$ & $59.9(9.1)$ & $74.1(8.1)$ & $58.5(9.0)$ \\
\hline \multicolumn{5}{|c|}{ Knee abduction $\left(^{\circ}\right)$} \\
\hline $\mathrm{ACL}_{\mathrm{R}}$ & $1.2(5.1)$ & $0.0(4.7)$ & $2.1(5.1)$ & $1.7(5.1)$ \\
\hline $\mathrm{ACL}_{\mathrm{PT}}$ & $4.1(5.5)$ & $3.4(6.3)$ & $5.7(4.1)$ & $4.8(5.2)$ \\
\hline CTRL & $2.3(5.0)$ & $1.6(6.2)$ & $2.0(4.9)$ & $0.8(5.1)$ \\
\hline \multicolumn{5}{|c|}{ Knee internal rotation $\left(^{\circ}\right)$} \\
\hline $\mathrm{ACL}_{\mathrm{R}}$ & $-0.1(7.0)$ & $0.4(6.8)$ & $1.4(6.8)$ & $2.8(6.7)$ \\
\hline $\mathrm{ACL}_{\mathrm{PT}}$ & $-1.5(5.5)^{\mathrm{a}}$ & $-1.6(6.7)^{\mathrm{a}}$ & $2.0(7.0)$ & $3.3(7.8)$ \\
\hline CTRL & $4.4(7.3)$ & $5.1(7.0)$ & $1.3(5.0)$ & $2.8(5.3)$ \\
\hline
\end{tabular}

Negative values in knee internal rotation denote external rotation. I/ND leg, injured leg for ACL groups and non-dominant leg for CTRL; NI/D leg, non-injured leg for ACL groups and dominant leg for CTRL; TO, Take-off phase; LA, Landing phase

a Significant within-subject difference observed to NI leg

to both $\mathrm{ACL}_{\mathrm{R}}$ and CTRL. Since an internal tibial torque also induces a coupled anterior tibial translation relative to the femur [13], this further supports a protective avoidance strategy of potentially risky knee positions for $\mathrm{ACL}_{\mathrm{PT}}$. Similar results of reduced knee sagittal plane movement for ACL-deficient persons compared to $\mathrm{ACL}_{\mathrm{R}}$ and CTRL groups have been shown in landings of a one-leg hop for distance [22]. This movement strategy seems not to be explained by quadriceps strength since no differences were shown between any of these groups for knee extension strength, although both treatment groups had lower knee flexor strength than controls. In contrast, in our study population only the concentric knee extension strength, but not concentric flexion strength, was lower for both $\mathrm{ACL}_{\mathrm{PT}}$ and $\mathrm{ACL}_{\mathrm{R}}$ compared to CTRL, as presented previously [26].

$\mathrm{ACL}_{\mathrm{R}}$ adapted a strategy where they increased trunk and hip flexion compared to both $\mathrm{ACL}_{\mathrm{PT}}$ and CTRL. Increased trunk flexion was indeed observed in both phases for the injured compared to the non-injured leg for $\mathrm{ACL}_{\mathrm{R}}$. Similar results with greater trunk flexion between legs for ACLreconstructed persons in another study further revealed a forward shift of the centre of pressure, which resulted in a more anterior position of the ground reaction force vector in relation to the hip, knee, and ankle joint axes [18]. This strategy shifted the joint moment from the knee to the adjacent joints. Instructions to land with increased trunk flexion also result in increased hip and knee flexion, but do not influence movement in the frontal or transversal planes, when compared to a preferred landing strategy during the vertical drop landing [4]. On the other hand, a landing strategy with a more upright trunk flexion results in increases in peak vertical ground reaction force, peak knee extensor moment, hip moment, and quadriceps amplitude [24]. A small knee flexion angle also increases the patellar tendon insertion angle and decreases the hamstrings insertion angle, which results in an increased anterior tibial shear force for a given anterior tibial shear load [4]. The $\mathrm{ACL}_{\mathrm{PT}}$ group would therefore seem to have adopted a movement strategy that restricts movement, although provokes an anterior tibial translation when compared to the other groups. This movement strategy was particularly evident when comparing their injured leg to the non-injured leg in Landing, demonstrating less knee flexion and knee internal rotation for the injured leg. In addition, the altered kinematics also for the non-injured leg in $\mathrm{ACL}_{\mathrm{PT}}$ compared to both $\mathrm{ACL}_{\mathrm{R}}$ and CTRL further indicate a crossover effect displayed to a greater extent than for $\mathrm{ACL}_{\mathrm{R}}$. The restrained movement strategy for $\mathrm{ACL}_{\mathrm{PT}}$ for both legs may indicate a greater vulnerability to challenging loadings of the knee in one-leg hops [21], possibly also increasing the risk of injury for the intact ACL of the other leg, and contributing to development of OA [2]. Of particular relevance here may be the average differences in knee rotation of $5.9^{\circ}$ in Takeoff and $6.7^{\circ}$ in Landing which were shown for $\mathrm{ACL}_{\mathrm{PT}}$ compared to CTRL for I/ND legs. These are above the value of $5^{\circ}$ shown to accelerate cartilage thinning [3], which may be detrimental to future knee health. Particular consideration should be given to the coupled average differences in knee flexion of $8.3^{\circ}$ in Take-off and $9.9^{\circ}$ in Landing between the same groups. For comparison, there were non-significant average values of knee flexion and knee rotation for $A C L_{R}$ and CTRL in Take-off of $2.3^{\circ}$ and $4.5^{\circ}$, respectively, and in Landing of $2.9^{\circ}$ and $4.7^{\circ}$, respectively. Hop tests with a greater emphasis on rotational demands may enhance this difference for $\mathrm{ACL}_{\mathrm{R}}$ compared to CTRL due to rotational instability that seems to remain after ACL reconstruction 

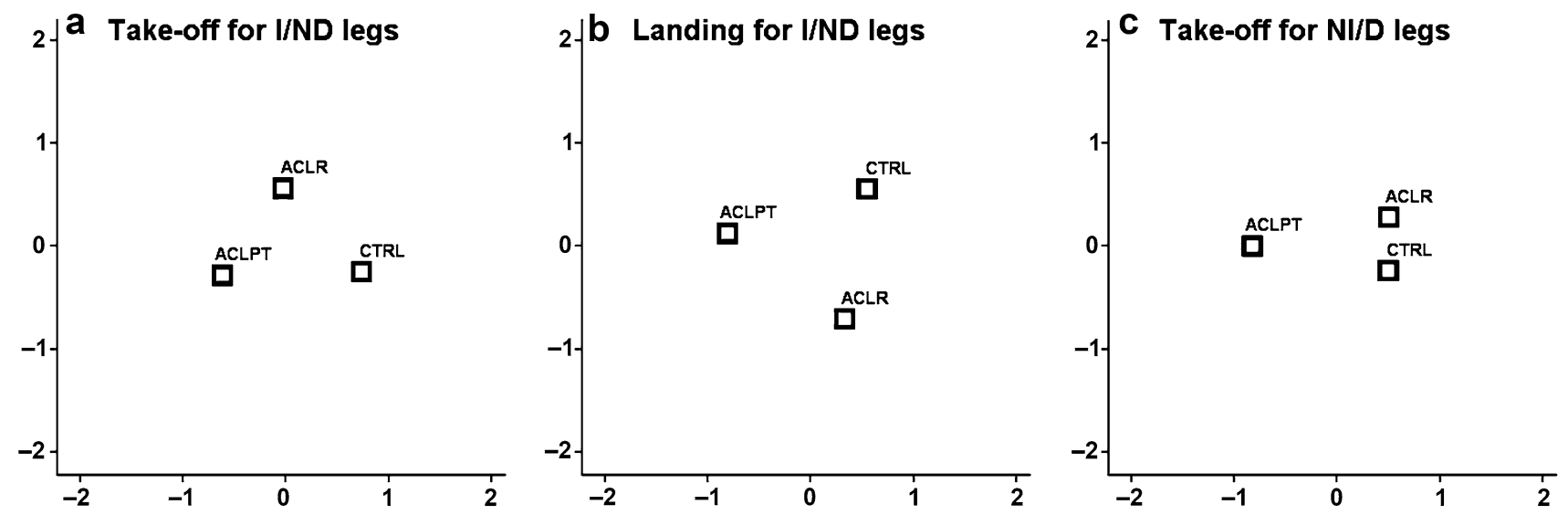

Fig. 3 Kinematic representation of discriminant function plots for all groups. Distances of group centroids on the $x$-axis and $y$-axis are of interest in $\mathbf{a}$ and $\mathbf{b}$, and the distance on the $x$-axis is of interest in c. Combinations of differences in kinematic variables between the groups correlate with these functions, thus separating the group centroids. These are in descending order with the highest correlation first: in a knee internal rotation and knee flexion contributes to dis- tances in $x$-axis, and trunk flexion, hip internal rotation, knee abduction, and hip flexion to distances in $y$-axis; in $\mathbf{b}$ knee flexion, hip flexion, knee internal rotation, and hip adduction contribute to distances in $x$-axis, and trunk flexion and hip internal rotation contribute to distances in $y$-axis; in c knee flexion, knee abduction, hip flexion, and hip internal rotation contribute to distances in $x$-axis

movement strategies certainly provides additional value, especially when evaluating treatment. A VH test is easy to administer in the clinic, is not time-consuming, enables comparisons of biomechanical asymmetries between injured and non-uninjured sides, and can be used to determine progress in rehabilitation. Different kinematics for both ACL groups are of clinical relevance since instability may exist irrespective of treatment. Both $\mathrm{ACL}_{\mathrm{PT}}$ and $\mathrm{ACL}_{\mathrm{R}}$ had $>90 \%$ LSI for hop height, despite demonstrating different movement patterns and low self-estimated knee function. Interpretation of results based on LSI scores or absolute measures from hop performances should therefore be analysed with caution when evaluating knee function if used without consideration of other knee function measures. Similar results have been found for ACL-injured persons achieving $>90 \%$ in dynamic tests despite presenting altered kinematics [19] or having self-estimated unstable knees [6, 30]. Hopefully, better and simpler motion analysis of movement strategies in the clinics may be developed based on more detailed analyses such as in the present study. Our results highlight the importance of incorporating the trunk and hip when evaluating movement strategies for ACL-injured persons in the clinics rather than only focusing on the knee joint.

Some limitations of the present study should be acknowledged. The use of skin markers implies risks of soft tissue artefacts influencing validity and reliability. To reduce this, clusters of markers were used on areas with large muscle groups for better approximations. Soft tissue artefacts seem to be similar between subjects and hence should not mask group differences [8]. Comparisons between the different 
treatment groups could also be questioned as this was not an RCT. Long-term kinematic studies are, however, very rare, and the biomechanical conditions from these cohorts were described without necessarily drawing direct conclusions about treatment recommendations. It should be mentioned that major improvements in $\mathrm{AC}$ reconstruction techniques has occurred during the past 20 years and need to be considered in relation to our results. Multiple health-related aspects over the years may also play a role, such as the precise impact and course of OA that may vary [17]. However, no effects of the degree of radiological knee OA on knee kinematics was found in an earlier report on the same study population [25]. The higher Tegner activity score for CTRL compared to both treatment groups could be questioned, but was partly explained by the high score assigned to occasional participation in downhill skiing during leisure time for many participants as they live in a northern climate. No group differences for IPAQ scores may indicate that the amount of activity does not explain the different movement patterns. Also, since BMI did not present any correlations with or influenced any of the kinematic results, the groups included in this study could be considered comparable for this biomechanical investigation.

\section{Conclusion}

This biomechanical study more than 20 years post-injury of the one-leg vertical hop during Take-off and Landing phases highlights different movement strategies for combinations of trunk, hip, and knee kinematics for the injured side of both ACL-reconstructed and ACL-deficient groups compared to CTRL and between ACL groups. Future controlled studies with stringent clinical and biomechanical assessment involving overall movement strategies of the trunk and leg post-ACL injury are desirable to evaluate type of treatment and course of rehabilitation and would be of relevance for clinical implementation.

Acknowledgements We would like to acknowledge Jonas Selling, research engineer, for helping with writing scripts and handling kinematic calculations, and Andrew Strong, Ph.D. student, for proofreading.

Authors' contributions JM carried out data and statistical analyses and drafted the manuscript. ET participated in data collection and helped to draft the manuscript. $\mathrm{CH}$ helped with data collection and to draft the manuscript. All authors read and approved the final manuscript.

\section{Compliance with ethical standards}

Conflict of interest The authors declare that they have no conflict of interest.
Funding Funding was provided by Swedish Scientific Council (Grant No. K2011-69X-21876-01-3), Young Researcher Awardee (Grant No. Dnr 223-514-09), Västerbotten County Council (Grant No. ALFVLL217301), Swedish National Centre for Research in Sports (Grant No. Dnr CIF 2013/5 P2014-0057), IngaBritt och Arne Lundbergs Research Foundation (Grant No. 363), and Umeå University School of Sport Science (Grant No. Dnr 3172).

Ethical approval All procedures performed in studies involving human participants were in accordance with the ethical standards of the institutional and/or national research committee and with the 1964 Helsinki declaration and its later amendments or comparable ethical standards.

Informed consent Informed consent was obtained from all individual participants included in the study.

Open Access This article is distributed under the terms of the Creative Commons Attribution 4.0 International License (http://creativecommons.org/licenses/by/4.0/), which permits unrestricted use, distribution, and reproduction in any medium, provided you give appropriate credit to the original author(s) and the source, provide a link to the Creative Commons license, and indicate if changes were made.

\section{References}

1. Alenezi F, Herrington L, Jones P, Jones R (2014) The reliability of biomechanical variables collected during single leg squat and landing tasks. J Electromyogr Kinesiol 24(5):718-721

2. Amis AA, Bull AMJ, Lie DTT (2005) Biomechanics of rotational instability and anatomic anterior cruciate ligament reconstruction. Oper Tech Orthop 15(1):29-35

3. Andriacchi TP, Briant PL, Bevill SL, Koo S (2006) Rotational changes at the knee after ACL injury cause cartilage thinning. Clin Orthop Relat Res 442:39-44

4. Blackburn JT, Padua DA (2008) Influence of trunk flexion on hip and knee joint kinematics during a controlled drop landing. Clin Biomech 23(3):313-319

5. Borgen FH, Seling MJ (1978) Uses of discriminant analysis following MANOVA: multivariate statistics for multivariate purposes. J Appl Psychol 63(6):689-697

6. Briem K, Ragnarsdottir AM, Arnason SI, Sveinsson T (2014) Altered medial versus lateral hamstring muscle activity during hop testing in female athletes 1-6 years after anterior cruciate ligament reconstruction. Knee Surg Sports Traumatol Arthrosc 24(1):12-17

7. Deneweth JM, Bey MJ, McLean SG, Lock TR, Kolowich PA, Tashman S (2010) Tibiofemoral joint kinematics of the anterior cruciate ligament-reconstructed knee during a single-legged hop landing. Am J Sports Med 38(9):1820-1828

8. Gao B, Zheng NN (2008) Investigation of soft tissue movement during level walking: translations and rotations of skin markers. J Biomech 41(15):3189-3195

9. Grip H, Häger C (2013) A new approach to measure functional stability of the knee based on changes in knee axis orientation. $\mathrm{J}$ Biomech 46(5):855-862

10. Grip H, Tengman E, Hager CK (2015) Dynamic knee stability estimated by finite helical axis methods during functional performance approximately twenty years after anterior cruciate ligament injury. J Biomech 48(10):1906-1914 
11. Gustavsson A, Neeter C, Thomee P, Silbernagel KG, Augustsson J, Thomee R, Karlsson J (2006) A test battery for evaluating hop performance in patients with an ACL injury and patients who have undergone ACL reconstruction. Knee Surg Sports Traumatol Arthrosc 14(8):778-788

12. Hewett TE, Myer GD, Ford KR, Heidt RS Jr, Colosimo AJ, McLean SG, van den Bogert AJ, Paterno MV, Succop P (2005) Biomechanical measures of neuromuscular control and valgus loading of the knee predict anterior cruciate ligament injury risk in female athletes: a prospective study. Am J Sports Med 33(4):492-501

13. Kanamori A, Zeminski J, Rudy TW, Li G, Fu FH, Woo SL (2002) The effect of axial tibial torque on the function of the anterior cruciate ligament: a biomechanical study of a simulated pivot shift test. Arthroscopy 18(4):394-398

14. Kellgren JH, Lawrence JS (1957) Radiological assessment of osteo-arthrosis. Ann Rheum Dis 16(4):494-502

15. Malfait B, Sankey S, Firhad Raja Azidin RM, Deschamps K, Vanrenterghem J, Robinson MA, Staes F, Verschueren S (2014) How reliable are lower-limb kinematics and kinetics during a drop vertical jump? Med Sci Sports Exerc 46(4):678-685

16. Markolf KL, Jackson SR, Foster B, McAllister DR (2014) ACL forces and knee kinematics produced by axial tibial compression during a passive flexion-extension cycle. J Orthop Res 32(1):89-95

17. Meunier A, Odensten M, Good L (2007) Long-term results after primary repair or non-surgical treatment of anterior cruciate ligament rupture: a randomized study with a 15-year follow-up. Scand J Med Sci Sports 17(3):230-237

18. Oberlander KD, Bruggemann GP, Hoher J, Karamanidis K (2013) Altered landing mechanics in ACL-reconstructed patients. Med Sci Sports Exerc 45(3):506-513

19. Orishimo KF, Kremenic IJ, Mullaney MJ, McHugh MP, Nicholas SJ (2010) Adaptations in single-leg hop biomechanics following anterior cruciate ligament reconstruction. Knee Surg Sports Traumatol Arthrosc 18(11):1587-1593

20. Ortiz A, Olson S, Libby CL, Trudelle-Jackson E, Kwon YH, Etnyre B, Bartlett W (2008) Landing mechanics between noninjured women and women with anterior cruciate ligament reconstruction during 2 jump tasks. Am J Sports Med 36(1):149-157
21. Pollard CD, Sigward SM, Powers CM (2010) Limited hip and knee flexion during landing is associated with increased frontal plane knee motion and moments. Clin Biomech 25(2):142-146

22. Roos PE, Button K, Sparkes V, van Deursen RW (2014) Altered biomechanical strategies and medio-lateral control of the knee represent incomplete recovery of individuals with injury during single leg hop. J Biomech 47(3):675-680

23. Ryan W, Harrison A, Hayes K (2006) Functional data analysis of knee joint kinematics in the vertical jump. Sports Biomech 5(1):121-138

24. Shimokochi Y, Ambegaonkar JP, Meyer EG, Lee SY, Shultz SJ (2013) Changing sagittal plane body position during singleleg landings influences the risk of non-contact anterior cruciate ligament injury. Knee Surg Sports Traumatol Arthrosc 21(4):888-897

25. Tengman E, Brax Olofsson L, Nilsson KG, Tegner Y, Lundgren L, Hager CK (2014) Anterior cruciate ligament injury after more than 20 years: I. Physical activity level and knee function. Scand J Med Sci Sports 24(6):491-500

26. Tengman E, Brax Olofsson L, Stensdotter AK, Nilsson KG, Hager CK (2014) Anterior cruciate ligament injury after more than 20 years. II. Concentric and eccentric knee muscle strength. Scand J Med Sci Sports 24(6):e501-e509

27. Tengman E, Grip H, Stensdotter A, Hager CK (2015) Anterior cruciate ligament injury about 20 years post-treatment: a kinematic analysis of one-leg hop. Scand J Med Sci Sports 25(6):818-827

28. VandenBerg C, Crawford EA, Sibilsky Enselman E, Robbins CB, Wojtys EM, Bedi A (2017) Restricted hip rotation is correlated with an increased risk for anterior cruciate ligament injury. Arthroscopy 33(2):317-325

29. Webster KE, Feller JA (2012) Tibial rotation in anterior cruciate ligament reconstructed knees during single limb hop and drop landings. Clin Biomech 27(5):475-479

30. von Porat A, Henriksson M, Holmstrom E, Thorstensson CA, Mattsson L, Roos EM (2006) Knee kinematics and kinetics during gait, step and hop in males with a 16 years old ACL injury compared with matched controls. Knee Surg Sports Traumatol Arthrosc 14(6):546-554 